\title{
P22 Bacteriophage Portal: The Conduit for DNA Packaging and Release
}

Jinghua Tang ${ }^{1}$, Gabriel C. Lander ${ }^{2}$, Adam Olia ${ }^{3}$, Rui $\mathrm{Li}^{4}$, Sherwood Casjens ${ }^{5}$, Peter Prevelige Jr. , Gino Cingolani ${ }^{6}$, John E. Johnson ${ }^{7}$, and Timothy S. Baker ${ }^{1,8}$

${ }^{1}$ Dept. of Chemistry \& Biochemistry, University of California, San Diego, La Jolla, CA 92093

${ }^{2}$ Life Science Division, Lawrence Berkeley National Lab, Berkeley, CA 94720

${ }^{3}$ Dept. of Biological Sciences, Purdue University, W. Lafayette, IN 47907

${ }^{4}$ Dept. of Microbiology, The University of Alabama, Birmingham, Birmingham, AL 35294

${ }^{5}$ Dept. of Pathology, University of Utah School of Medicine, Salt Lake City, UT 84112

${ }^{6}$ Dept. of Biochemistry \& Molecular Biology, Thomas Jefferson University, Philadelphia, PA 19107

${ }^{7}$ Dept. of Molecular Biology, The Scripps Research Institute, La Jolla, CA 92037

${ }^{8}$ Division of Biological Sciences, University of California, San Diego, La Jolla, CA 92093

P22 (family Podoviridae) is a model system for studying particle assembly and DNA packaging in bacteriophage. Mature virions contain nine structural proteins with 415 copies of the major capsid protein, gp5, assembling into a nearly icosahedral, $\mathrm{T}=7$ laevo, quasi-symmetric head. A 12-fold symmetric dodecamer of gp1 ("portal") occupies one of the vertices of the icosahedron and forms an axial channel through which the dsDNA genome enters and exits the head. The portal also serves as the attachment site for the tail machine, formed by gp4, gp9, gp10, and gp26, each of which is an oligomer with respective subunit stoichiometries in a 12:18:6:3 ratio. Three ejection proteins (gp7, gp16, and gp20) are packaged inside the head and assist DNA release during infection of the host bacterium (Salmonella). Earlier cryo-reconstructions of the whole (asymmetric) virions at 17-20 resolution $[1,2]$ revealed the overall morphology of the phage and the symmetry-mismatch between the head and tail components.

Careful reanalysis of CCD images of $\sim 22,000$ mature P22 virions (FIG 1A) has enabled us to compute a markedly improved asymmetric reconstruction at 7.8 - $\AA$ resolution (FIG $1 \mathrm{~B}, \mathrm{C}$ ). The atomic model for the full-length (725 a.a.) portal [3] closely fits the virion density map, especially for the core part of the portal (a.a. 1-601) (FIG 1C,D). The 150- $\AA$ long, coiled-coil, barrel structure redefines the scabbard-like tube of density (previously interpreted as ejection proteins [2]) that extends from the portal core to near the center of the head (FIG 1C,D). This barrel adopts a distinctly different conformation in the virion compared to that in crystals. In the virion, the bundle of twelve alpha-helices that form the barrel is twisted and compressed slightly inwards towards the axis of the barrel near its base. This concerted rearrangement shifts the distal end of the barrel $\sim 10 \AA$ towards the portal core. Such rearrangements in the barrel structure are likely induced by increased pressure exerted by the DNA as it fills the head during packaging, and this may enable the barrel to act as a valve to entrap the DNA until the tail machine assembles.

The X-ray model of the core-gp4 complex [3] also nicely fit the virion density map and revealed the presence of an unexpected interaction among the extended C-terminus of gp4 (a.a. 130-150) and the portal and capsid proteins (FIG 1E). This stretch of gp4 residues lies wedged between the capsid and portal and serves to anchor gp4 and the rest of tail machine securely to the head [4].

\section{References}

[1] Chang, J., et al., Structure 14 (2006) 1073.

[2] Lander, G. C., et al., Science 312 (2006) 1791.

[3] Olia, A. S., et al., Nat. Struct. Mol. Biol. (2011) in press. 
[4] Research supported by National Institutes of Health grants R01 GM054076 (JEJ), R37 GM033050 and 1S10 RR-020016 (TSB), AI074825 (SC), and R56 AI076509 (GC). Image data were recorded at the National Resource for Automated Molecular Microscopy at TSRI, which is supported by the NIH through the National Center for Research Resources' P41 program (RR17573).
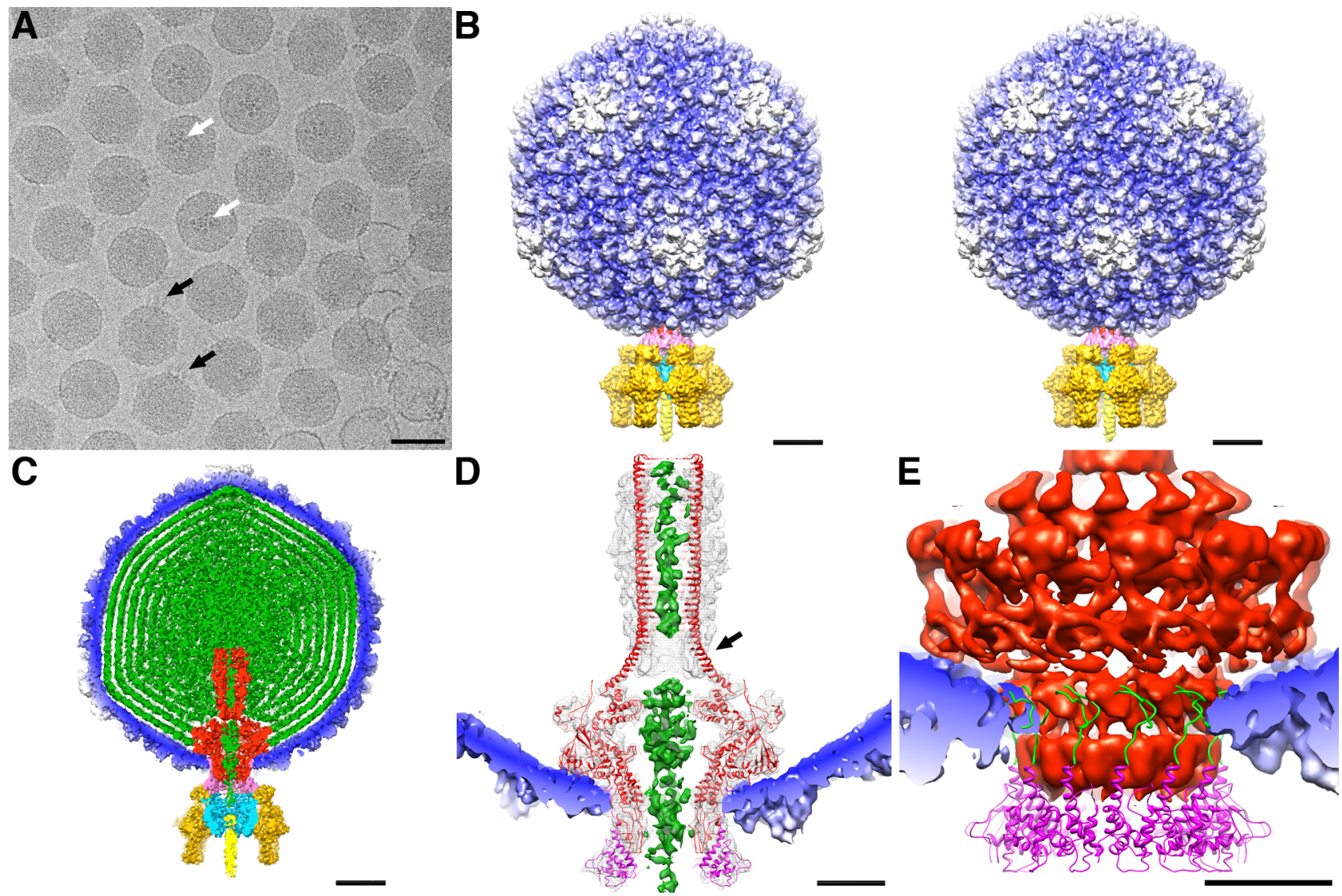

FIG 1. P22 virion structure. (A) CCD image of purified suspension of P22 virions (Scale bar $=50$ $\mathrm{nm}$ ). Many tails can be seen in side (black arrows) and end on (white arrows) views. (B) Stereo view of a segmented and color-coded 3D density map of the virion (Scale bar $=10 \mathrm{~nm}$; color coding identified in next panel). (C) Planar cross-section ( 6-nm thick) through the center of the virion shown in (B) highlighting individual components: gp1 (red), gp4 (pink), gp5 (color-cued radially from inner (blue) to outer (white) radii), gp9 (dark yellow), gp10 (cyan), gp26 (yellow), and DNA plus pilot proteins (green). Scale bar $=10 \mathrm{~nm}$. (D) Close-up view of a thin slab of the virion density map highlighting the portal/gp4 complex (grey mesh) wedged inside the capsid (blue, solid density), the DNA (green, solid density) contained within the portal channel, and a ribbon model of the portal/gp4 crystal structure (gp1 and gp4 in red and magenta, respectively) fitted into density attributed to portal/gp4. The base (proximal end) of the barrel portion of the gp1 crystal structure clearly veers outside the density (black arrow). Scale bar $=5 \mathrm{~nm}$. (E) Side view of the portal density (red) and the fitted gp4 crystal structure (magenta ribbon model). The C-terminal loop portion of gp4 (a.a. 122-150) is highlighted in green and extends up along the outer surface of the portal core and lies wedged between portal and capsid subunits (solid blue density). Scale bar $=5 \mathrm{~nm}$. 